\title{
Audit of Caesarean Sections in a Single Centre: Analysis of its Frequency and Indications
}

\author{
Tasneem Akhtar, ${ }^{1}$ Kainat Sarwar, ${ }^{1}$ Sadia Shakeel, ${ }^{1}$ Noreena Saba ${ }^{1}$
}

\begin{abstract}
Background: Caesarean section serves a substantial parameter of comprehensive emergency obstetric and neonatal care and it is a package of care that groups together basic emergency obstetric care as well.

Objective: To evaluate the frequency and indications of caesarean section in a tertiary care hospital.

Methodology: This cross-sectional study was conducted in the department of Obstetrics and Gynaecology, Bahawal Victoria Hospital, Bahawalpur from June to November 2018. The records of all the women delivering either vaginally or by caesarean section during the study period were retrieved. Age, socioeconomic status, parity, type of delivery, and indications for which caesarean sections was done. Data was analyzed by SPSS version 20.

Results: A total of 4575 deliveries were conducted during the study period. Out of these, 2605 (57\%) had caesarean sections, and 1970 (43\%) delivered vaginally. Maternal age ranged from 20 to 40 years. The most common reason for caesarean section was repeat caesarean section (22\%) followed by fetal distress $(21 \%)$.

Conclusion: This study revealed that there is a high frequency of caesarean section in tertiary care hospital, and the most common reasons for caesarean section were repeat caesarean section and fetal distress. The departmental protocols and SOPs must be made for indications of caesarean section. Adherence to standard guidelines and protocols for managing labor is required.
\end{abstract}

Keywords: Caesarean section, Audit, Indications

Article Citation: Akhtar T, Sarwar K,Shakeel S, Saba N. Audit of Caesarean Sections in a Single Centre: Analysis of its Frequency and Indications, JSZMC 2021;12(3):03-07. DOI: https://doi.org/10.47883/jszmc.v12i20

This Open Access Article in Journal of Sheikh Zayed Medical College is licensed under a Creative Commons Attribution- 4.0 International License(CC BY 4.0).

\section{Introduction}

Caesarean section is an essential component of comprehensive emergency obstetric and neonatal care (CEmONC), a care bundle that includes basic emergency obstetric care i.e., parenteral antibiotics, anticonvulsants, uterotonic agents, manual removal of placenta, manual vacuum aspiration, neonatal resuscitation, assisted vaginal delivery and blood transfusion, in addition to these basic care services anesthesia and caesarean section are included. ${ }^{1}$

Regarding clinical audit, its a process of quality improvement that aims to improve patient care and outcomes through systemic review of care against standard criteria over a period of time followed by implementation of change if any. ${ }^{2}$ It is vital to improving obstetric care on a priority basis especially in our and other low resource countries where there are still high maternal and fetal morbidities. ${ }^{3}$ Caesarean section is an intervention where either vaginal delivery is contraindicated or not possible. It is a life-saving option where maternal and/or fetal life are in danger. Where a lot beneficial there are certain hazards associated with caesarean section. A major health problem is the undue caesarean section which is associated with significant risk to both mother and baby. Not only that it also extends the effect to the future reproductive health of the woman. In addition to these, undue caesarean sections pose an extra workload to the health professionals and a raised financial burden on the patient's pocket. ${ }^{4,5,6}$ Based on the national population survey rate of caesarean delivery in Pakistan is around $25 \%$ with a range of $35 \%$ to $21 \%$.

Caesarean section in the developed countries is almost 20\% (15-25\%), with less than 1:10,000 maternal mortality. ${ }^{8,9}$ Although fruitful when really indicated, malpractice of undue caesarean sections have put forward much health hazards and economical burden. ${ }^{10}$ Caesarean section has significantly raised maternal and perinatal morbidity. While comparing maternal mortality in women undergone caesarean section to those having

1. Department of Gynaecology and Obstetrics, Bahawal Victoria Hospital, Bahawalpur, University of Health Sciences, Lahore, Pakistan.

Correspondence: Dr. Kainat Sarwar, Assistant Professor, Department of Gynaecology and Obstetrics, Bahawal Victoria Hospital,

Bahawalpur. Pakistan.

Email: ksarwar61@gmail.com

Received: 06-07-2020

Published: 20-09-2021 
vaginal deliveries, maternal morbidity is 5-10 times higher. The caesarean scar also has an effect on future mode of delivery. ${ }^{11,12}$

No consensus is available on the rate of caesarean section. WHO states that the rate of caesarean section is more than $12-15 \%$ is of no added benefits. ${ }^{13}$ In South East Asia China has the maximum caesarean section rate. ${ }^{14}$ In the USA caesarean section rate is around $38 \%{ }^{15}$ The caesarean trend is becoming an epidemic and all can foresee the effects of this increase in terms of maternal and fetal outcomes both in developed and developing countries. The objective of this study was to evaluate the frequency and indications of caesarean section in a tertiary care hospital.

\section{Methodology}

After taking approval from the ethical committee of the institute, a cross-sectional study was conducted in the department of Obstetrics and Gynaecology, Bahawal Victoria Hospital Bahawalpur. Six month data were collected from June to November 2018 of all the women delivering either vaginally or by caesarean section. The data of the patient's deficient related to study variables was excluded. Frequency and indications for which caesarean sections was noted. Demographic data i.e, age, socioeconomic status and parity were recorded. Data was analyzed by SPSS version 20 .

\section{Results}

In this descriptive study, women who delivered either vaginally or by caesarean section were included in this study. There were 4575 deliveries in a six-month period, of these 2605 (57\%) had caesarean sections (Figure-I) and 1970 (43\%) delivered vaginally. Range of maternal age was from 20 to 40 years. Most of the patients belonged to lower or middle socioeconomic status $(89 \%)$. The most common reason for caesarean section was repeat caesarean section $(22 \%)$ followed by fetal distress (21\%). Failed progress of labor and antepartum hemorrhage (APH), placenta previa, and abruption were both $13 \%$ each. Fetal malpresentation was observed in $7 \%$ of cases (Table-I).
Figure-I: Frequency of Caesarean Section $(n=4575)$

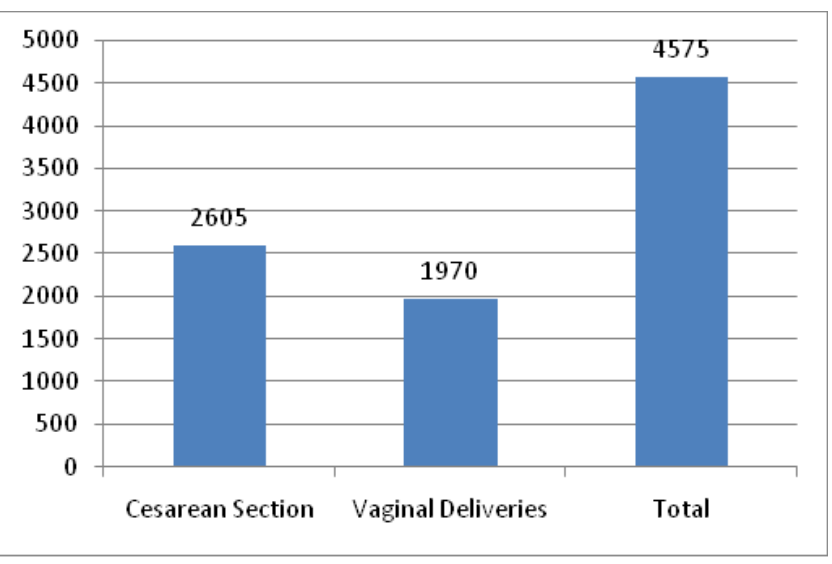

Table-I: Indications of Caesarean Section

\begin{tabular}{|l|c|}
\hline Indication of caesarean section & No (\%) \\
\hline Repeat caesarean section & $566(22 \%)$ \\
\hline Fetal distress & $558(21 \%)$ \\
\hline Failed progress of labour & $342(13 \%)$ \\
\hline APH (placenta previa and abruption) & $331(13 \%)$ \\
\hline Hypertensive disorders PIH \& Eclampsia & $225(8 \%)$ \\
\hline Malpresentation & $194(7 \%)$ \\
\hline Cephalo-pelvic disproportion & $77(3 \%)$ \\
\hline Post term pregnancy & $64(2 \%)$ \\
\hline IUGR & $58(2 \%)$ \\
\hline Bad obstetric history & $50(2 \%)$ \\
\hline Multiple pregnancy & $43(1.6 \%)$ \\
\hline Gestational diabetes mellitus & $42(1.6 \%)$ \\
\hline Precious pregnancy & $35(1.3 \%)$ \\
\hline Obstructed labour & $20(0.8 \%)$ \\
\hline Total caesarean sections & 2605 \\
\hline
\end{tabular}

\section{Discussion}

High caesarean section rate is an area of concern because of the fact for caesarean section in itself poses negative effects on mother and child health, and literature shows a high risk of future medical complications to both. ${ }^{16,17}$ Additionally a normal delivery after the previous caesarean section gets risky, and high fertility coupled with a high caesarean section rate would lead to additional risk of multiple major surgeries.

Moreover in an already resource-constrained community repeated surgical interventions will lead to more health care spending. The reasons for high caesarean section rate may be beyond medical causes such as financial gains of the healthcare providers or medically unnecessary caesarean section on women's own choice.

Caesarean section rate has climbed up both in 
developed and developing countries over the last three to four decades i.e., 6-7\% in 1970 to $24-30 \%$ in $2003 .{ }^{16}$ In our study, caesarean section rate was comparatively very high $(57 \%)$ other local studies had $20 \%$ and $60 \%$ caesarean delivery rates. ${ }^{17,18}$ Similar to our study another local study in which caesarean section rate was $56 \%$ was noted ${ }^{19}$ and also a study conducted in Brazil showed a similar rate of caesarean section. ${ }^{20}$ While in our study women usually belonged to a lower socioeconomic class it was different in some other locally performed studies in which more caesareans were done in upper class women. ${ }^{17-19}$ Caesarean section was done more widely in women of upper socioeconomic when compared to women of lower social class. This was noted in two studies from the USA and England..$^{20,21}$ Repeat caesarean section was the most frequent $(22 \%)$ indication of the caesarean section in our study, which is in accordance with many other studies done on caesarean sections. ${ }^{22,23}$

According to another study previous caesarean section is an indication of repeat caesarean section in almost $20 \%{ }^{24}$ This high incidence of repeat caesarean section is due to lesser planned vaginal birth after caesarean (VBAC) in most departments. Incidence of $\mathrm{VBAC}$ varies in different setup usually from $10 \%$ to $90 \%{ }^{25}$ In our set up mostly women present in advanced labor so a careful watch is kept on the progress of labor and vaginal births are successfully conducted in patients with previous caesarean scars. For this reason rate of VBAC after one caesarean section is higher in our setup. Rate of successful VBAC in previous one caesarean scar is almost $76 \%{ }^{26}$

Following the repeat caesarean section, the next most frequent indication of caesarean sections in our set was fetal distress and reduces fetal movements by the mother (21\%). Much importance is given to the subjective feeling of the mother about reduced fetal movements in our setup.

There is a lack of standard clinical criteria about fetal distress thus leading to a higher number of caesareans. Electronic fetal heart rate monitoring is routinely done in our setup and other hospitals as well. Any change in fetal heart rate that is not supported by the change in $\mathrm{pH}$ of fetal blood may lead to a low threshold of caesarean section. Fetal blood $\mathrm{pH}$ to see fetal acidosis is not routinely done in our setup so CTG interpretations may become doubtful and thus adding to caesarean section. Caesarean section done on account of this indication can be lessened if fetal heart rate changes are augmented by fetal $\mathrm{pH}$ as well. ${ }^{27}$ Another very frequent indication of caesarean section in our study was failed induction and progress of labour (13\%). This indication was seen more in primigravida patients when the cervix is not favorable. ${ }^{28,29}$

Haver Kamp and colleagues and Leveno and coworkers stated a higher rate of caesarean section when fetal monitoring was done using electronic fetal monitoring as compared to when intermittent auscultation was done. ${ }^{30}$ Malpresentations including breech presentation was responsible for $7 \%$ of caesarean section rate in our study. Caesarean sections being done for breech presentation is an aspect of modern obstetrics. In some countries, the rate of caesarean for breech has raised to $80 \%$. This affects the overall rate of caesarean sections and also increased the chance of repeat caesarean sections in a future pregnancy. ${ }^{31}$

Hypertensive disorder and eclampsia also contribute much to rate of caesarean section $(8 \%)$. Other indications that accounted $28 \%$ of caesarean sections included APH, both placenta previa and placental abruption, multiple pregnancies, post-term pregnancies, diabetes, precious pregnancy, CPD, and bad obstetric history. In Lyari General Hospital, Karachi, and Isra Medical University, Hyderabad Miscellaneous causes of caesarean section were 20\% and $64 \%$ respectively. ${ }^{31,32}$ Critical appraisal is required to identify the cause in cases where indications are not clear i.e, poor bishop score, reduced fetal movements, on-demand caesarean and unspecified reasons. Over time on demand caesareans have put on an increased burden of caesareans on the hospitals. It means that the patient herself demands elective caesarean section without any valid indication although such indication is not mentioned in our study.

More and more work is being done on reducing complications of caesarean sections, but there is a need to work on preventing this rapid rise in caesarean section rate. In this study, it is noted that lack of audit system for caesarean sections on regular basis has resulted in poor documentation and recordkeeping. Unnecessary caesareans can be prevented by proper justifiable indications for caesarean, audit, and clinical governance. 


\section{Conclusion}

This study revealed that there is a high frequency of caesarean section in our tertiary care hospital, and most common reasons for caesarean section were repeat caesarean section and fetal distress. The departmental SOPs at gynecology and obstetric units must be made for justifiable indications of caesarean section. Awareness and promotion of public health and health education regarding risks of caesarean and benefits of vaginal delivery. Motivational talks and classes for the antenatal population to deliver vaginally. Antenatal counseling and pre labor classes to increase the pain threshold. Proper booking and referral system. Proper nutrition and supplements must be given to increase maternal health. Regular audit of caesarean section rates and indications must be maintained and well documented. Adherence to standard guidelines and protocols for managing labor is required.

Authors Contribution: TA: Design of work and drafting. KSH: Acquisition of data and drafting. SS: Interpretation of data and revising. NS: Conception of work and revising.

All authors critically revised and approve its final version.

Conflict of Interest: Author has declared no conflict of interest.

Sources of Funding: The source of funding was self.

\section{Disclaimer: None}

\section{References}

1. WHO, UNFPA, UNICEF, AMDD. Monitoring emergency obstetric care. 2009: Sexual and Reproductive Health. Geneva.

2. Wylie BJ, Mirza FG. Caesarean delivery in the developing world. Clinics in Perinatology 2008: 35: 571582.

3. Graham W, Wagaarachi P, Penney G, Binns MC, Antwi KY. Criteria for clinical audit of the quality of hospitalbased obstetric care in developing countries. Reproductive Health 2000: 78:5.

4. Souza JP, Gulmezoglu A, Lumbiganon P, Laopaiboon M, Carroli G. Caesarean section without medical indications is associated with an increased risk of adverse short-term maternal outcomes: 2004-2008 WHO global survey on maternal and perinatal health. BMC Medicine 2010: 8: 71.

5. Villar J, Carroli G, Zavaleta N, Donner A, Wojdyla D. Maternal and neonatal individual risks and benefits associated with caesarean delivery: Multicentre prospective study. BMJ 2007: 335: 1025.
6. Ronsmans C, Holtz S, Stanton C. Socio-economic differentials in caesarean rates in developing countries: A retrospective analysis. Lancet 2006: 368: 1516-1523.

7. C-section rates around globe at epidemic level. The Associated Press; msn Canadian 2010 . Available at http://www.msnbc.msn.com/id/34826186.

8. Betrán AP, Merialdi M, Lauer JA, Bing-Shun W, Thomas J, Van Look P. Rates of caesarean section: analysis of global, regional and national estimates. Paediatr Perinat Epidemiol 2007;21(2):98-113

9. Lee SI, Khang YH, Lee Ms. Women attitude towards mode of delivery in South Korea: A society with high caesarean section rates. Birth 2004;31(2);108-16

10. Petrou S, Henderson J, Glazener C. Economic aspects of caesarean section and alternative modes of delivery. Best Pract Res ClinObstetGynaecol. 2001;15:145-63.

11. Yang Q, Wen SW, Oppenheimer L, Chen XK. Association of caesarean delivery for first birth with placenta praevia and placental abruption in second pregnancy. BJOG.2007;114:609-13.

12. Onwere C, Gurol-Urganci I, Cromwell DA. Maternal morbidity associated with placenta praevia among women who had elective caesarean section.Eur J Obstet Gynecol Reprod Biol. 2011; 159:62-66.

13. Sweet RD, Ledger WJ. Puerperal infectious morbidity. Am J Obstet Gynaecol 1973; 117:1093-100.6. World Health Organization: Appropriate technology for birth. Lancet 1985,:326(8452):436-37.

14. Lumbiganon P, Laopaiboon M, Gülmezoglu AM. Method of delivery and pregnancy outcomes in Asia: the WHO global survey on maternal and perinatal health 2007-08. Lancet .2010;375:490-99.

15. Barber EL, Lundsberg LS, Belanger K, Christian M. Indications contributing to the increasing caesarean delivery rate. Obstet Gynecol 2011;118:29-38

16. Christilaw JE. Caesarean section by choice: constructing a reproductive rights framework for the debate. International J Gynae/Obs 2006: 94:262-68.

17. Ali L, Tayyab S. Caesarean section rate: current trends. J Surg Pakistan 2007; 12:64-66.

18. Choudhary SM, Ayaz A. Efforts to reduce Caesarean Section Rate. J Surg Pakistan 2003; 8:25-27.

19. Jasia Jabeen, Muhammad Hassaan Mansoor, Ammarah Mansoor. Analysis of Indications of Caesarean Sections. JRMC; 2013;17(1):101-103.

20. Belizan JM, Althabe F, Barros FC. Rates and implications of caesarean sections in Latin America: Ecological study. BMJ 1993;319:1397-1400.

21. Wagner M. Born in the USA: How a broken maternity system must be fixed to put women and children first. 1 edition University of California Press; 2006.

22. Barley K, Bottle A, Jarman B. Social class and elective caesareans in the English NHS. Br Med J 2004; 328:13991403.

23. Belgrave S. Caesarean section. NZ FamPhysic 2003; 30:26972. 
24. Sheikh L, Tehseen S, Gowani SA, Bhurgri H. Reducing the rate of primary caesarean section - an audit. J Pak Med Assoc 2008; 58:444-48.

25. Haider G, Zehra N, Munir AA, Haider A. Frequency and indication of caesarean section in a tertiary care hospital. Pak J Med Sci 2009;25(5)791-96.

26. Guise JM, Berli M, McDonagh M, Osterwell P. Safety of vaginal birth after caesarean section: a systematic review. Obstetrics and Gynaecology 2004; 103:420-29

27. Tang CH, Wang HI. Risk-adjusted Caesarean Section rate for the assessment of physician performance. BMC Public Health 2006, 6: 246-49.

28. Rayburn WF. Minimising the risk from elective induction of labour. J Reprod Med 2007; 52: 671-76.
29. Dabbas M, Al-Sumadi A. Caesarean Section rate: much room for reduction. Clin Exp Obstet Gynecol. 2007; 34: $146-48$.

30. Haverkamp AD, Thompson HE, Mcfee JG. The evaluation of continuous fetal heart rate monitoring in high-risk pregnancy. Am J Obstetrics Gynaecology 1976; 125:310-20.

31. Gulfareen H, Nishat Z, Aftab AM, AmbreenH. Caesarean section rate. Pak J Med Sci 2009; 25:786-91.

32. Karim F, Ghazi A, Ali T, Aslam R. Trends and determinants of caesarean section. Journal of Surgery Pakistan (International) 2011; 16 (1):22-27 